\title{
Geleitwort zur zweiten Auflage
}

Der Textteil dieser jetzt vorliegenden verbesserten und wesentlich erweiterten 2. Auflage ist umfangreicher geworden als in der 1. Auflage. Das erforderte die Fülle des vorliegenden Materials, das weite Kreise der papierverarbeitenden Industrie ansprechen wird.

Es ist ein Grunderfordernis bei der Herausgabe eines Fachbuches, an die ständig fortschreitende Technik zu denken, diese möglichst erschöpfend für die Praxis zu behandeln und im Interesse der Leser auszuwerten.

Ein Fachbuch soll den Fachmann ansprechen, der ständig mit den Techniken vertraut sein muß. Wie vielseitig gerade das Gebiet der Papierveredelung ist, geht $u$. a. hervor aus der Buchbesprechung eines in den USA erschienenen kleinen Werkes, die mir freundlicherweise 1962 die Schriftleitung der Allgemeinen Papier-Rundschau zur Verfügung stellte. Ich möchte deren Wortlaut allen Lesern dieses Buches zur Kenntnis geben:

"Auf einem Raum von etwa 100 Seiten liegt hier eine sehr anschaulich und volkstümlich abgefaßte Schrift vor, die sich zum Ziel gesetzt hat, das Wissen um unseren Werkstoff Papier, seine Erzeugung, Veredelung - etwa durch Beschichten mit Kunststoffen - und Verarbeitung in weite Kreise zu tragen. Hier scheint uns ein gutes Beispiel dafür vorzuliegen, wo man wieder einmal von den Amerikanern lernen kann, für den eigenen Werkstoff, sein Werden und seinen Wert im Rahmen der gesamten Volkswirtschaft werbend vor dem Forum der breiten Offentlichkeit zu sprechen.

Wir könnten uns denken, daß ein solches, auf die deutschen Verhältnisse abgestelltes Informationsbuch auch durchaus im Interesse unseres Faches liegen dürfte."

Als Herausgeber dieser 2. Auflage der Papierveredelung möchte ich behaupten, daß sie berufen sein dürfte, eine Lücke der Fachliteratur auszufüllen und gemäß dem Schlußabsatz der kritischen Betrachtung mit gleichen Worten als Informationsbuch, auch auf die deutschen Verhältnisse abgestellt, durchaus im Interesse unseres Faches liegen dürfte“.

- 'Paper, the fifth wonder' (Papier, das fünfte Wunder). Thomas Publishing Company, 724 Desnoyer Street, Kaukana, Wis., USA. Preis \$4.- zuzüglich Porto. 
Das IV. Kapitel dürfte besonders aufschlußreich sein für die Gestaltung ideenreicher und werbewirksamer Erzeugnisse der schmückenden Industrie. Es hat an Aktualität noch gewonnen durch zwei weitere Beiträge, die zur Vervollständigung des Themas wesentlich sind.

Kunst und Technik in der reproduzierenden Industrie sind nicht voneinander zu trennen. Daher werden Gedanken und Anregungen zu neuem Schaffen stets erwünscht sein, nicht nur für den Fachmann in der Praxis, sondern auch für die Gestalter selbst, für die Kreise also, welche berufsmäßig die maltechnischen Vorlagen schaffen, nach denen dann die Industrie produziert und die sehr zahlreichen Erzeugnisse in Form und Farbe gestaltet, die aus unserem Alltagsleben nicht mehr fortzudenken sind.

Bei der textlichen Gestaltung dieser Auflage wurde wiederum auf die Behandlung der manuellen künstlerischen Techniken Wert gelegt. Einige der in diesem Buch behandelten Techniken sind manuell und handwerklich im eigentlichen Sinne des Wortes. Es ist gut, wenn der berufliche Nachwuchs diese auch theoretisch kennenlernt. Die Theorie ist stets die Vorstufe für die Praxis.

Bewußt habe ich die rein handwerklichen Techniken zu Wort kommen lassen. Unsere Zeit ist mit ihrer Schnellebigkeit und der damit verbundenen ständigen Rationalisierung $\mathrm{zu}$ neuen, immer wertvolleren Veredelungsmethoden des Papiers übergegangen. Sie machte aus dem künstlerischen Handwerker den Spezialisten einer modernen Industrie. Es erscheint mir wichtig, gerade für den beruflichen Nachwuchs auch die alten handwerklichen Techniken $\mathrm{zu}$ beschreiben.

Die schmückenden Industrien - Druck-Kolorit-Farbspritzung - sowie die Herstellung der Monogramme und das Prägen, insbesondere die Reliefprägungen fanden stets mein besonderes Interesse in allen Stadien ihres Entstehens und insbesondere in ihrer Bedeutung für die Produktion.

Einen größeren Raum als in der 1. Auflage umfassen in dieser Ausgabe die "Glückwunschkarten" (Kapitel V). Diese sind nicht mehr fortzudenkende, für den Fachhändler lohnende Verkaufsartikel.

Die Mode stagniert nicht und auch die Ansprüche der Käuferkreise an die Motivwahl und Ausstattung steigern sich. Durch die fortschreitende Entwicklung im Laufe der Zeit waren einige wesentliche Ergänzungen dieses Kapitels erforderlich, bedungen allein schon durch den sich stets wandelnden Publikumsgeschmack.

Im Rahmen der Artikelserie über die Glückwunschkarten, behandelt von namhaften Fachleuten sowie vorwiegend vom Herausgeber selbst, wird ersichtlich: Diese Verkaufsartikel, hergestellt in großen Sortimenten, sind unvermindert als marktgängig anzusehen. 
Es handelt sich um Erzeugnisse der Kleinkunst. Die geschmackvolle Auswahl der Motive für die bildhaften Darstellungen in Verbindung mit der künstlerischen Ausgestaltung ist ein wichtiger Faktor bei der Herstellung, vorwiegend durch die Nutzbarmachung der modernen Stahlstichprägung.

Bei den Glückwunschkarten spielt nicht nur die bildliche Motivwahl, sondern hauptsächlich die Beschriftung eine wesentliche Rolle, eine dankbare Aufgabe für die Nutzbarmachung der Stahlstichprägung, die als Ausstattungstechnik hoch im Kurs steht. Es sei besonders auf die das Thema erschöpfende Arbeit aus der Feder eines bekannten Fachmannes über die Stahlstichprägungen hingewiesen (Kapitel VII).

Die vorwiegend durch die Stahlstichprägung geschaffenen Ausstattungsmöglichkeiten sind sehr vielseitig, vor allem durch die Auswahl der verschiedenfarbigen, in der Anwendung recht wirkungsvollen Lackfarben. Nicht unerwähnt seien auch die künstlerisch gravierten Prägestempel mit ihren neuzeitlichen Schriftgarnituren, die allen Ansprüchen der Käufer Rechnung tragen.

Im X. Kapitel wird über neuzeitliche Maschinen zur Veredelung durch Beschichten berichtet, das inhaltlich besonders instruktiv ist. Es dürfte die Fachinteressenten für diese Sparte interessieren. Die technischen Beschreibungen werden durch zahlreiche Abbildungen im Tafelanhang unterstützt.

Am Schluß des vorgenannten Kapitels finden wir noch die Beschreibungen von zwei weiteren neuen Maschinen zur Papierverdelung, nämlich einer Folienklebemaschine, die bei Herausgabe des Buches noch in der Konstruktion befindlich war, und einer Hochleistungs-Beschichtungs- und Heißklebemaschine.

Der technische Teil des Buches bringt als XI. Kapitel einen aufschlußreichen Beitrag über das Lackieren, Kalandrieren und Glanzfoliieren. Diese Arbeitsgebiete werden eingehend von einem bekannten Fachmann auf diesem Sondergebiet dargestellt auf Grund sachkundiger Kenntnis. Sie dürfen als eine wertvolle Ergänzung der zahlreichen Möglichkeiten für die Veredelung des Papiers zu betrachten sein.

Ich hoffe, daß zu den bisherigen Lesern noch weitere kommen werden, insbesondere von Berufsfachleuten sowie auch seitens des Nachwuchses, aus dem sich erst noch ein neuer Stamm von Facharbeitern für die papierverarbeitende Industrie entwickeln soll.

Den im Text genannten und ungenannten Mitarbeitern danke ich für ihre mir gegebenen wertvollen Anregungen durch textliche Verbesserungen, Ergänzungen und Bereitstellung geeigneten fachlichen Materials.

Meinem früheren und jetzigen Mitarbeiter an dieser Auflage, Herrn Johs. Kirstein, Hamburg, danke ich besonders für seine wertvollen Anregungen. 
Auch den Fachfirmen gebührt mein Dank, die mir Abbildungen von Maschinen und Geräten für den Tafelanhang zur Verfügung stellten, da dieses Anschauungsmaterial wertvoll ist für den Leser des Buches, eine Tatsache, auf die in den Kritiken bei Erscheinen meiner Fachbücher in der einschlägigen Fachpresse immer wieder hingewiesen wurde.

Berlin 1965

WALTER HESS 